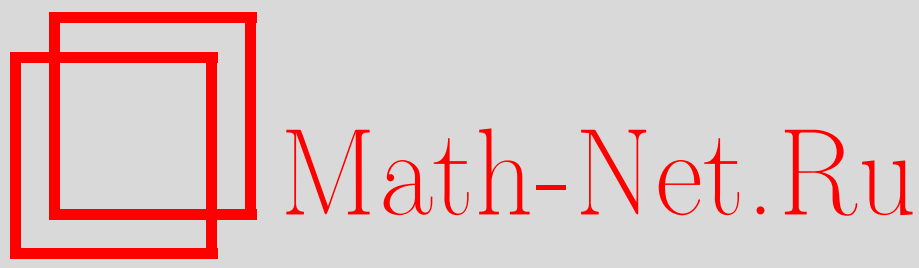

Н. Н. Пустовойтов, Ортопоперечники некоторых классов периодических функций двух переменных с заданной мажорантой смешанных модулей непрерывности, Изв. РАН. Сер. матем., 2000, том 64, выпуск 1, 123-144

DOI: https://doi.org/10.4213/im276

Использование Общероссийского математического портала Math-Net.Ru подразумевает, что вы прочитали и согласны с пользовательским соглашением

http: //www. mathnet.ru/rus/agreement

Параметры загрузки:

IP: 34.229 .108 .108

26 апреля 2023 г., 18:35:10 
УДК 517.518 .8

\author{
Н. Н. Пустовойтов
}

\title{
Ортопоперечники некоторых классов периодических функций двух переменных с заданной мажорантой смешанных модулей непрерывности
}

\footnotetext{
В работе рассматриваются ортопоперечники классов периодических функций двух переменных с заданной мажорантой смешанных модулей непрерывности порядка $l$, содержащей, кроме степенных, также и логарифмические множители. В ряде случаев найдены точные порядки ортопоперечников указанных классов функций.

Библиографиия: 9 наименований.
}

\section{§1. Введение. Формулировки теорем}

В настоящей работе изучаются ортопоперечники, определяемые для класса функций $F \subset L_{p}$ равенством

$$
d_{M}^{\perp}\left(F, L_{p}\right)=\inf _{\left\{u_{i}(\mathbf{x})\right\}_{i=1}^{M}} \sup _{f \in F}\left\|f(\mathbf{x})-\sum_{i=1}^{M}\left(f(\cdot), u_{i}(\cdot)\right) u_{i}(\mathbf{x})\right\|_{p},
$$

где inf берется по ортонормированным системам $\left\{u_{i}(\mathbf{x})\right\}_{i=1}^{M}$ ограниченных функций.

Впервые ортопоперечники для классов Соболева и Никольского были рассмотрены В. Н. Темляковым в [1]. Затем они изучались В. Н. Темляковым [2]-[4], Динь Зунгом [5] и Э. М. Галеевым [6].

Мы рассмотрим классы периодических функций двух действительных переменных с мажорантой смешанных модулей непрерывности более общего вида, чем для классов Никольского. Мы используем методы, аналогичные методам, примененным в работах [1]-[3].

Как видно из определения ортопоперечников, приближением функции служит ее ортогональная проекция на подпространство размерности $M$.

Kaк и в работах [2], [3], рассмотрим тесно связанные с ортопоперечниками величины

$$
d_{M}^{B}\left(F, L_{p}\right)=\inf _{G \in \mathscr{L}_{M}(B)_{p}} \sup _{f \in F}\|f(\mathbf{x})-G f(\mathbf{x})\|_{p}
$$

где $B \geqslant 1, \mathscr{L}_{M}(B)_{p}$ - множество линейных операторов $G$, в область определения $D(G)$ которых входят все тригонометрические полиномы, а множество значений имеет размерность не выше $M$ и содержится в пространстве $L_{p}\left([-\pi ; \pi]^{2}\right)$ и для всех $\mathbf{k}=\left(k_{1}, k_{2}\right)\left(k_{j}\right.$ целые $)$ выполнено неравенство

$$
\left\|G e^{i(\mathbf{k}, \mathbf{x})}\right\|_{2} \leqslant B
$$

(C) Н.н. Пустовойтов, 2000 
Операторы ортогонального проектирования на подпространства размерности $M$ содержатся в $\mathscr{L}_{M}(1)_{2}$.

Из определения величин $d_{M}^{B}\left(F, L_{p}\right)$ и $d_{M}^{\perp}\left(F, L_{p}\right)$ следует неравенство

$$
d_{M}^{B}\left(F, L_{p}\right) \leqslant d_{M}^{\perp}\left(F, L_{p}\right)
$$

Очевидно, что эти величины не меньше, чем поперечники по Колмогорову. Однако в отличие от поперечников по Колмогорову для ортопоперечников в ряде случаев легче указать подпространства, даюшие наилучшие (в смысле ортопоперечников) приближения. Кроме того, даже в том случае, когда известно оптимальное (в смысле поперечника по Колмогорову) подпространство, довольно трудно указать элемент наилучшего приближения. В случае же ортопоперечника, если известно оптимальное подпространство, элемент наилучшего приближения определяется явно как ортогональная проекция на это подпространство.

Будем рассматривать функции двух действительных переменных $f(\mathbf{x})=$ $f\left(x_{1}, x_{2}\right)$, имеюшие период $2 \pi$ по каждой переменной.

Для рассматриваемых функций введем разности порядка $l$ :

$$
\Delta_{\mathbf{h}}^{l} f(\mathbf{x})=\Delta_{\left(h_{1}, h_{2}\right)}^{l} f\left(x_{1}, x_{2}\right)=\Delta_{h_{1}}^{l} \Delta_{h_{2}}^{l} f\left(x_{1}, x_{2}\right),
$$

т.е. берется разность порядка $l$ с шагом $h_{j}$ по переменной $x_{j}, j=1,2$.

Эти разности порождают модуль непрерывности порядка $l$ :

$$
\Omega(f ; \mathbf{t})_{q}=\Omega\left(f ;\left(t_{1}, t_{2}\right)\right)_{q}=\sup _{\substack{\left|h_{j}\right| \leqslant t_{j} \\ j=1,2}}\left\|\Delta_{\mathbf{h}}^{l} f(\mathbf{x})\right\|_{q}
$$

$\Phi$ ункция $\Omega(f ; \mathbf{t})_{q}$ по каждой переменной $t_{j}$ обладает всеми свойствами одномерного модуля непрерьвности порядка $l$.

Рассмотрим классы функций

$$
H_{q}^{\Omega}=\left\{f(\mathbf{x}) \in L_{q}^{0}\left([-\pi, \pi]^{2}\right): \Omega(f ; \mathbf{t})_{q} \leqslant \Omega(\mathbf{t})\right\}
$$

Здесь условие $f(\mathbf{x}) \in L_{q}^{0}\left([-\pi, \pi]^{2}\right)$ означает, что $f(\mathbf{x}) \in L_{q}\left([-\pi, \pi]^{2}\right)$ и, кроме того,

$$
\int_{-\pi}^{\pi} f(\mathbf{x}) d x_{j}=0, \quad j=1,2
$$

Функция $\Omega(\mathbf{t})=\Omega\left(t_{1}, t_{2}\right)$ - заданная функция типа смешанного модуля непрерьвности порядка $l$. Это означает, что она обладает свойствами:

1) $\Omega\left(t_{1}, t_{2}\right)$ непрерывна при $t_{j} \geqslant 0, j=1,2$;

2) $\Omega\left(t_{1}, t_{2}\right)$ возрастает по каждой переменной;

3) $\Omega(0,0)=\Omega\left(t_{1}, 0\right)=\Omega\left(0, t_{2}\right)=0$;

4) $\Omega\left(k_{1} t_{1}, k_{2} t_{2}\right) \leqslant k_{1}^{l} k_{2}^{l} \Omega\left(t_{1}, t_{2}\right), k_{j}$ целье, $j=1,2$.

В работах [3], [4] исследованы ортопоперечники классов Никольского, т.е. классов $H_{q}^{\Omega}$ с мажорантой $\Omega(\mathbf{t})=\Omega\left(t_{1}, \ldots, t_{d}\right)=\prod_{j=1}^{d} t_{j}^{r_{j}}$. 
Мы же рассмотрим функцию $\Omega(\mathbf{t})$ вида

$$
\begin{array}{ll}
\Omega(\mathbf{t})=\Omega\left(t_{1}, t_{2}\right)=\frac{t_{1}^{r}}{\left(\log \frac{1}{t_{1}}\right)^{b_{1}}} \cdot \frac{t_{2}^{r}}{\left(\log \frac{1}{t_{2}}\right)^{b_{2}}}, & 0<t<1, \\
\Omega(0,0)=\Omega\left(t_{1}, 0\right)=\Omega\left(0, t_{2}\right)=0, & 0<r<l .
\end{array}
$$

Здесь и дальше рассматриваются логарифмы по основанию 2 . Так как $r<l$, то $\Omega(\mathbf{t})$ обладает всеми свойствами смешанного модуля непрерывности порядка $l$ (указанные вьше свойства 1)-4)). В отличие от классов Никольского, здесь вводятся логарифмические множители. При $b_{1}<r$ и $b_{2}<r$ влияние этих множителей невелико и они "не нарушают" изотропность классов $H_{q}^{\Omega}$, т.е. параметры $b_{1}$ и $b_{2}$ равноправны (см. теоремы 1 и 2 ). В случаях же $b_{1} \leqslant r, b_{2}>r ; b_{1}>r, b_{2} \leqslant r$; $b_{j}>r, j=1,2$, влияние логарифмических множителей велико и возникает анизотропность. Эта анизотропность порождает эффект "не своих" гиперболических крестов. Впервые этот эффект отметил С. А. Теляковский [7]. Для анизотропных классов Никольского $H_{q}^{\mathbf{r}}, \mathbf{r}=\left(r, \ldots, r, r_{\nu+1}, \ldots, r_{d}\right), r<r_{\nu+1} \leqslant \cdots \leqslant r_{d}$, вместо "своих" гиперболических крестов (определение $\rho(\mathbf{s})$ см. ниже)

$$
Q_{n}^{\mathbf{r}}=\bigcup_{(\boldsymbol{\gamma}, \mathbf{s}) \leqslant n} \rho(\mathbf{s}), \quad \mathbf{r}=r \boldsymbol{\gamma}, \quad \boldsymbol{\gamma}=\left(1, \ldots, 1, \gamma_{\nu+1}, \ldots, \gamma_{d}\right),
$$

лучше брать "не свои" гиперболические кресты $Q_{n}^{\mathbf{r}^{\prime}}=\bigcup_{\left(\boldsymbol{\gamma}^{\prime}, \mathbf{s}\right) \leqslant n} \rho(\mathbf{s})$, где $\mathbf{r}^{\prime}=r \boldsymbol{\gamma}^{\prime}$, $\gamma^{\prime}=\left(1, \ldots, 1, \gamma_{\nu+1}^{\prime}, \ldots, \gamma_{d}^{\prime}\right)$, причем $1<\gamma_{j}^{\prime}<\gamma_{j}, j=\nu+1, \ldots, d$.

Дело в том, что хотя $Q_{n}^{\mathbf{r}^{\prime}}$ шире, чем $Q_{n}^{\mathbf{r}}$, количество элементов “своего" гиперболического креста по порядку совпадает с количеством элементов "не своего" гиперболического креста, однако приближение функции тригонометрическими полиномами с гармониками из "не своих" гиперболических крестов по порядку лучше. Такой же эффект наблюдается и в нашем случае (см. теоремы 5-8), т.е. не только различие степенных порядков дает эффект "не своих" гиперболических крестов, но и определенное различие в показателях логарифмов.

Функция, заданная равенством (2), удовлетворяет условиям $(\mathrm{S})$ и $\left(\mathrm{S}_{l}\right)$ (см. [8], [9]). Определим эти свойства. Функция одного переменного $h(\tau)$ обладает свойством (S), если существует $0<\alpha<1$, при котором $h(\tau) / \tau^{\alpha}$ почти возрастает, т.е. существует $C>0$, не зависящее от $\tau$, такое, что

$$
\frac{h\left(\tau_{1}\right)}{\tau_{1}^{\alpha}} \leqslant C \frac{h\left(\tau_{2}\right)}{\tau_{2}^{\alpha}}, \quad 0<\tau_{1} \leqslant \tau_{2}<1 .
$$

Будем говорить, что функция $\Omega(\mathbf{t})$ обладает свойством (S), если она обладает этим свойством по каждой переменной при условии, что другая переменная фиксирована.

Функция одной переменной $h(\tau)$ обладает свойством $\left(\mathrm{S}_{l}\right)$, если существует $0<\beta<l$, при котором отношение $h(\tau) / \tau^{\beta}$ почти убывает, т.е. сушествует $C>0$, не зависящее от $\tau$, при котором

$$
\frac{h\left(\tau_{1}\right)}{\tau_{1}^{\beta}} \geqslant C \frac{h\left(\tau_{2}\right)}{\tau_{2}^{\beta}}, \quad 0<\tau_{1} \leqslant \tau_{2}<1 .
$$

Свойство $\left(\mathrm{S}_{l}\right)$ для функции $\Omega(\mathbf{t})$ определяется аналогично свойству $(\mathrm{S})$.

Так как $\Omega(\mathbf{t})$ из $(2)$ обладает свойствами $(\mathrm{S})$ и $\left(\mathrm{S}_{l}\right)$, то для рассматриваемого класса $H_{q}^{\Omega}$ верна следуюшая теорема о представлении [9]. 
ТЕОрема А. Периодическая функиия $f(\mathbf{x})$ из пространства $L_{q}^{0}\left([-\pi ; \pi]^{2}\right)$ принадлежит классу $H_{q}^{\Omega}$ c $\Omega(\mathbf{t})$ из (2) в том и только том случае, если для векторов $\mathbf{s}=\left(s_{1}, s_{2}\right)$ с натуральными компонентами справедливы неравенства

$$
\begin{aligned}
\left\|\delta_{\mathbf{s}}(f ; \mathbf{x})\right\|_{q} \ll \Omega\left(2^{-\mathbf{s}}\right), & 1<q<\infty \\
\left\|A_{\mathbf{s}}(f ; \mathbf{x})\right\|_{q} \ll \Omega\left(2^{-\mathbf{s}}\right), & 1 \leqslant q \leqslant \infty
\end{aligned}
$$

При $1<q<\infty$ эти условия эквивалентны, $2^{-\mathrm{s}}=\left(2^{-s_{1}}, 2^{-s_{2}}\right)$. Здесь

$$
\delta_{\mathbf{s}}(f ; \mathbf{x})=\sum_{\mathbf{k} \in \rho(\mathbf{s})} \hat{f}(\mathbf{k}) e^{i(\mathbf{k}, \mathbf{x})}
$$

-двоичная пачка ряда Фурье функции $f(\mathbf{x})$ (определение $\rho(\mathbf{s})$ см. ниже); $A_{\mathbf{s}}(f ; \mathbf{x})$ обозначает свертку $\delta_{\mathbf{s}}(f ; \mathbf{x})$ с ядром

$$
A_{\mathbf{s}}(\mathbf{x})=4 \prod_{j=1}^{2}\left(V_{2^{s_{j}-1}}\left(x_{j}\right)-V_{2^{s_{j}-2}}\left(x_{j}\right)\right),
$$

порожденным ядрами Валле-Пуссена $V_{m}(\tau)$ порядка $2 m-1$ (считаем $\left.V_{2^{-1}}(\tau) \equiv 1\right)$, T.e.

$$
A_{\mathbf{s}}(f ; \mathbf{x})=\delta_{\mathbf{s}}(f ; \mathbf{x}) * A_{\mathbf{s}}(\mathbf{x})
$$

ЗАмечание 1. Достаточность в теореме А означает, что если для функции $f(\mathbf{x})$ верны неравенства (3) либо (4), то существует число $B>0$, при котором $f_{1}(\mathbf{x})=B f(\mathbf{x}) \in H_{q}^{\Omega}$.

Нам понадобится следуюшая

ТЕорема (Литтлвуда-Пэли). Если $f(\mathbf{x}) \in L_{p}^{0}\left([-\pi ; \pi]^{2}\right)$, то для $1<p<\infty$

$$
\|f(\mathbf{x})\|_{p} \ll\left\|\left(\sum_{\mathbf{s}}\left|\delta_{\mathbf{s}}(f ; \mathbf{x})\right|^{2}\right)^{1 / 2}\right\|_{p} \ll\|f(\mathbf{x})\|_{p} .
$$

Здесь сумма берется по всем векторам $\mathbf{s}=\left(s_{1}, s_{2}\right)$ с натуральными $s_{1}$ и $s_{2}$.

Из теоремы Литтлвуда-Пэли вытекает

СлеДСтвиЕ. Для $f(\mathbf{x}) \in L_{p}^{0}\left([-\pi ; \pi]^{2}\right)$ при $1<p<\infty$ верно неравенство

$$
\|f(\mathbf{x})\|_{p} \ll\left(\sum_{\mathbf{s}}\left\|\delta_{\mathbf{s}}(f ; \mathbf{x})\right\|_{p}^{p_{0}}\right)^{1 / p_{0}}, \quad p_{0}=\min \{p, 2\}
$$

При доказательстве оценок сверху нам понадобится следуюшая лемма, доказанная В.Н. Темляковым в [2, с. 25]. 
ЛЕмма А. Для $1 \leqslant q<p<\infty$ и для $f(\mathbf{x}) \in L_{q}^{0}\left([-\pi ; \pi]^{2}\right)$ верно неравенство

$$
\|f(\mathbf{x})\|_{p}^{p} \ll \sum_{\mathbf{s}}\left(\left\|\delta_{\mathbf{s}}(f ; \mathbf{x})\right\|_{q} 2^{\|\mathbf{s}\|_{1}(1 / q-1 / p)}\right)^{p} .
$$

Здесь сумма берется по всем векторам $\mathbf{s}$ с натуральными координатами, $\|\mathbf{s}\|_{1}$ равна сумме координат вектора. Определим некоторые множества, порожденные линиями уровня функции $\Omega(\mathbf{t})$.

Для вектора $\mathbf{s}=\left(s_{1}, s_{2}\right)$ с натуральными компонентами определим двоичную пачку

$$
\rho(\mathbf{s})=\left\{\mathbf{k}=\left(k_{1}, k_{2}\right): k_{j} \text { целые, } 2^{s_{j}-1} \leqslant\left|k_{j}\right|<2^{s_{j}}, \quad j=1,2\right\} .
$$

Для натурального $N$ положим

$$
\varkappa(N)=\left\{\mathbf{s}=\left(s_{1}, s_{2}\right): s_{j} \in \mathbb{N}, \quad j=1,2, \quad \Omega\left(2^{-\mathbf{s}}\right) \geqslant \frac{1}{N}\right\},
$$

где $2^{-\mathbf{s}}=\left(2^{-s_{1}}, 2^{-s_{2}}\right)$. Далее, пусть

$$
\varkappa^{\perp}(N)=\mathbb{N}^{2} \backslash \varkappa(N) .
$$

В качестве приближающих подпространств возьмем множества тригонометрических полиномов, спектр которых лежит во множествах

$$
Q(N)=\bigcup_{\mathbf{s} \in \varkappa(N)} \rho(\mathbf{s})
$$

В этой работе речь идет о порядковых равенствах и порядковых неравенствах. Так, запись $A(N) \ll B(N)$ означает, что существует величина $C>0$, не зависящая от $N$ и такая, что $A(N) \leqslant C B(N)$. Порядковое равенство $A(N) \asymp B(N)$ означает, что $C^{-1} A(N) \leqslant B(N) \leqslant C A(N)$, где $C>0$ не зависит от $N$.

Наряду с множествами $Q(N)$ рассмотрим также множества

$$
\Gamma(N)=\left\{\mathbf{k}=\left(k_{1}, k_{2}\right):\left|k_{j}\right| \in \mathbb{N}, \quad j=1,2, \quad \Omega\left(\frac{1}{\left|k_{1}\right|}, \frac{1}{\left|k_{2}\right|}\right) \geqslant \frac{1}{N}\right\},
$$

т.е.

$\Gamma(N)=\left\{\mathbf{k}=\left(k_{1}, k_{2}\right):\left|k_{j}\right| \in \mathbb{N}, \quad j=1,2, \quad\left|k_{1}\right|^{r}\left(\log \left|k_{1}\right|\right)_{+}^{b_{1}}\left|k_{2}\right|\left(\log \left|k_{2}\right|\right)_{+}^{b_{2}} \leqslant N\right\}$,

где

$$
(\log \tau)_{+}= \begin{cases}\log \tau, & \tau=2,3, \ldots \\ 1, & \tau=1 .\end{cases}
$$

Множества $Q(N)$ и $\Gamma(N)$ эквивалентны, а именно из неравенств

$$
\Omega\left(\left|k_{1}\right|^{-1},\left|k_{2}\right|^{-1}\right) \leqslant \Omega\left(2^{1-s_{1}}, 2^{1-s_{2}}\right) \leqslant 2^{2 l} \Omega\left(2^{-\mathbf{s}}\right) \leqslant 2^{2 l} \Omega\left(\left|k_{1}\right|^{-1},\left|k_{2}\right|^{-1}\right),
$$

справедливых для $\mathbf{k}=\left(k_{1}, k_{2}\right) \in \rho(\mathbf{s})$, вытекает, что

$$
\Gamma(N) \subset Q\left(2^{2 l} N\right) \subset \Gamma\left(2^{2 l} N\right) .
$$

Далее будет показано, что число элементов $|\Gamma(N)|$ множества $\Gamma(N)$ имеет степенной порядок роста (см. утверждение 1$)$. В силу этого, а также вьшеуказанного вложения имеем $|Q(N)| \asymp|\Gamma(N)|$.

Для числа элементов множества $\Gamma(N)$ справедливо 
УТВЕРЖДЕНИЕ 1. Число әлементов множества $Г(N)$ по порядку равно:

$$
|\Gamma(N)| \asymp N^{1 / r} \Phi\left(b_{1}, b_{2}, r, N\right)
$$

əдe

$$
\Phi\left(b_{1}, b_{2}, r, N\right)= \begin{cases}(\log N)^{-b_{1} / r-b_{2} / r+1}, & b_{1}<r, \quad b_{2}<r ; \\ (\log N)^{-b_{2} / r} \log \log N, & b_{1}=r, \quad b_{2} \leqslant r ; \\ (\log N)^{-b_{1} / r} \log \log N, & b_{1}<r, \quad b_{2}=r ; \\ (\log N)^{-b_{1} / r}, & b_{1} \leqslant r, \quad b_{2}>r ; \\ (\log N)^{-b_{2} / r}, & b_{1}>r, \quad b_{2} \leqslant r ; \\ (\log N)^{-b / r}, & b_{1}>r, \quad b_{2}>r, \quad b=\min \left\{b_{1}, b_{2}\right\} .\end{cases}
$$

В дальнейшем нам понадобятся множества

$$
\theta(N)=\left\{\mathbf{s}=\left(s_{1}, s_{2}\right): s_{j} \in \mathbb{N}, \quad j=1,2, \quad \frac{1}{2^{l} N} \leqslant \Omega\left(2^{-\mathbf{s}}\right)<\frac{1}{N}\right\} .
$$

Замечание 2. Множество $\theta(N)$ непусто. Действительно, пусть $\mathbf{s}=\left(s_{1}, s_{2}\right)$ такое, что $s_{j} \in \mathbb{N}, j=1,2, \mathbf{s} \in \varkappa(N)$, a $\left(s_{1}, s_{2}+1\right) \notin \varkappa(N)$. Предположим, что $\left(s_{1}, s_{2}+1\right) \notin \theta(N)$, т.е. $\Omega\left(2^{-s_{1}}, 2^{-s_{2}}\right)<1 /\left(2^{l} N\right)$. Тогда, учитывая то, что $\mathbf{s} \in \varkappa(N)$, и свойство 4$)$ функции $\Omega(\mathbf{t})$, получим

$$
\frac{1}{N} \leqslant \Omega\left(2^{-s_{1}}, 2^{-s_{2}}\right) \leqslant 2^{l} \Omega\left(2^{-s_{1}}, 2^{-\left(s_{2}+1\right)}\right)<\frac{1}{N} .
$$

Противоречие. Это показывает, что $\theta(N)$ непусто, а именно для каждого $s_{1}$ такого, что $\mathbf{s}=\left(s_{1}, s_{2}\right) \in \varkappa(N)$, сушествует $\tilde{s}_{2} \in \mathbb{N}$, при котором $\left(s_{1}, \tilde{s}_{2}\right) \in \theta(N)$.

Для числа элементов множества $\theta(N)$ верно

УТВЕРЖДЕНИЕ 2. Имеем

$$
|\theta(N)| \asymp \log N
$$

Сформулируем теоремы об ортопоперечниках и величинах $d_{M}^{B}\left(H_{q}^{\Omega}, L_{p}\right)$. Рассмотрим вначале случай $b_{1}<r, b_{2}<r$. В этом случае положим $M=|Q(N)|$, откуда следует, что

$$
\begin{aligned}
M & \asymp N^{1 / r}(\log N)^{-b_{1} / r-b_{2} / r+1}, \\
N & \asymp M^{r}(\log M)^{b_{1}+b_{2}-r}, \quad \log M \asymp \log N .
\end{aligned}
$$


ТЕОРЕМА 1. Пусть функиия $\Omega(\mathbf{t})$ задана формулой (2) с

$$
r>\left(\frac{1}{q}-\frac{1}{p}\right)_{+} \equiv \max \left\{0 ; \frac{1}{q}-\frac{1}{p}\right\}
$$

Пусть, далее, $b_{j}<r, j=1,2$. Предположим, что $1 \leqslant p \leqslant \infty, 1 \leqslant q \leqslant \infty, u$ при $1 \leqslant q<p<\infty$ будем сиитать, что $b_{j}<\frac{r}{p(1 / q-1 / p)}$. Тогда

$$
d_{M}^{B}\left(H_{q}^{\Omega}, L_{p}\right) \asymp M^{-r+(1 / q-1 / p)}+(\log M)^{-b_{1}-b_{2}+r+h(p, q)} .
$$

Здеcь

$$
h(p, q)= \begin{cases}1-1 / q, & p=\infty, \quad 1 \leqslant q \leqslant \infty \\ 2 / p-1 / q, & 1 \leqslant q<p<\infty \\ 1 / p, & 1<q=p \leqslant 2 \\ 1 / 2, & 1 \leqslant p \leqslant q, \quad q \geqslant 2, \quad p<\infty \\ 1 / q, & 1 \leqslant p \leqslant q \leqslant 2 .\end{cases}
$$

ТЕОРема 2. Пусть $\Omega(\mathbf{t})$ определена равенством $(2), r>(1 / q-1 / p)_{+}$, $1 \leqslant q, \quad p \leqslant \infty,(p, q) \neq(1,1),(\infty, \infty) ; b_{j}$ удовлетворяют условиям теоремы 1. Тогда

$$
d_{M}^{\perp}\left(H_{q}^{\Omega}, L_{p}\right) \asymp M^{-r+(1 / q-1 / p)}+(\log M)^{-b_{1}-b_{2}+r+h(p, q)},
$$

где величина $h(p, q)$ определена $(11)$.

В теоремах 1 и 2 оптимальными (в смысле порядка) подпространствами являются подпространства тригонометрических полиномов с гармониками из множеств $Q(N)$.

Пусть, далее, $b_{1}>r, b_{2}=r$. В этом случае результаты не такие полные, как в теоремах 1 и 2 . При таких $b_{1}$ и $b_{2}$ будет $(M=|Q(N)|)$

$$
\begin{gathered}
M \asymp N^{1 / r}(\log N)^{-b_{1} / r} \log \log N, \\
N \asymp M^{r}(\log M)^{b_{1}}(\log \log M)^{-r} .
\end{gathered}
$$

ТЕОРема 3. Пусть функиия $\Omega(\mathbf{t})$ задается равенством (2) с $r>(1 / q-$ $1 / p)_{+} u b_{1}<r, \quad b_{2}=r$. Предположим также, что параметры $p$ u $q$ удовлетворяют одному из соотношений: $p=\infty, q=1 ; p=2, q=1$; $1 \leqslant p \leqslant q, q \geqslant 2, \quad p<\infty ; 1 \leqslant p \leqslant q \leqslant 2$. Тогда

$$
d_{M}^{B}\left(H_{q}^{\Omega}, L_{p}\right) \asymp M^{-r+(1 / q-1 / p)}+(\log M)^{-b_{1}+h(p, q)}(\log \log M)^{r},
$$

где величина $h(p, q)$ определена (11). 
ТЕорема 4. Пусть $\Omega(\mathbf{t})$ и параметры $p, q u b_{j}, \quad j=1,2$, удовлетворяют условиям теоремы $3,(p, q) \neq(1,1)$. Тогда

$$
d_{M}^{\perp}\left(H_{q}^{\Omega}, L_{p}\right) \asymp M^{-r+(1 / q-1 / p)}+(\log M)^{-b_{1}+h(p, q)}(\log \log M)^{r}
$$

с величиной $h(p, q)$, определенной в (11).

В теоремах 3 и 4 оптимальные (в смысле порядка) подпространства - это подпространства тригонометрических полиномов с гармониками из $Q(N)$.

Далее перейдем к случаю $b_{1} \leqslant r, b_{2}>r$. В этом случае будет $(M=|Q(N)|)$

$$
M \asymp N^{1 / r}(\log N)^{-b_{1} / r}, \quad N \asymp M^{r}(\log M)^{b_{1}} .
$$

Рассмотрим здесь, кроме множеств $Q(N)$, также множества $Q^{\prime}(N)=$ $\bigcup_{\mathbf{s} \in \varkappa^{\prime}(N)} \rho(\mathbf{s})$, где $\varkappa^{\prime}(N)=\left\{\mathbf{s}=\left(s_{1}, s_{2}\right): s_{j} \in \mathbb{N}, j=1,2,2^{r s_{1}} s_{1}^{b_{1}} 2^{r s_{2}} s_{2}^{b_{2}^{\prime}} \leqslant N\right\}$, причем $r<b_{2}^{\prime}<b_{2}$. Множества $Q^{\prime}(N)$ играют роль "не своих" гиперболических крестов. Как видно из утверждения 1 ,

$$
\left|Q^{\prime}(N)\right| \asymp|Q(N)| \asymp N^{1 / r}(\log N)^{-b_{1} / r} .
$$

Как легко убедиться, множество $Q^{\prime}(N)$ шире, чем $Q(N)$.

TEорема 5. Пусть функиия $\Omega(\mathbf{t})$ задана равенством (2) с $r>(1 / q-1 / p)_{+}$ u $b_{1} \leqslant r, \quad b_{2}>r$. Пусть $1 \leqslant p \leqslant \infty, \quad 1 \leqslant q \leqslant \infty$. Предположим, что при $p=\infty, \quad 1 \leqslant q<\infty$

$$
\frac{b_{2}}{r}\left(\frac{2}{q}-1\right)>1, \quad \frac{b_{2}}{r} \cdot \frac{1}{q}>1
$$

Пусть, далее, при $1 \leqslant q<p<\infty$

$$
\frac{b_{2}}{r} \cdot \frac{1}{q}>1, \quad \frac{b_{2}}{r}\left(\frac{2}{q}-1\right)>1, \quad \frac{b_{2}}{r}\left(p^{\prime}-1\right)>1, \quad \frac{b_{2}}{r}\left(\frac{p}{q}-1\right)>1 .
$$

Предположим также, что $b_{2}>r+1 / q_{0} \quad\left(q_{0}=\min \{q, 2\}\right)$ nри $1 \leqslant p \leqslant q<\infty$, a $b_{2}>r+1$ в случае $q=\infty$. Тогда

$$
d_{M}^{B}\left(H_{q}^{\Omega}, L_{p}\right) \asymp M^{-r+(1 / q-1 / p)_{+}}(\log M)^{-b_{1}} .
$$

ТеОрема 6. Пусть выполнены условия теоремы $5,(p, q) \neq(1,1),(\infty, \infty)$. Тогда

$$
d_{M}^{\perp}\left(H_{q}^{\Omega}, L_{p}\right) \asymp M^{-r+(1 / q-1 / p)_{+}}(\log M)^{-b_{1}} .
$$


ЗАмечАниЕ 3 . Фактически теоремы 5 и 6 не охватывают все пары $(p, q), 1 \leqslant$ $p \leqslant \infty, 1 \leqslant q \leqslant \infty$. Так, из первого неравенства (12) следует, что $1 \leqslant q<2$. Вообще говоря, с учетом $1 \leqslant q<2$ из первого неравенства (12) следует второе. Что касается неравенств (13), то пара $p=2, q=1$, очевидно, удовлетворяет им. И чем больше $b_{2}$, тем шире диапазон допустимых пар $(p, q)$.

Оптимальными в смысле порядка подпространствами являются подпространства тригонометрических полиномов со спектром из $Q(N)$ при $q<p$ и из $Q^{\prime}(N)$ при $p \leqslant q$.

Положим, далее, $b_{1}>r, b_{2}>r, b=\min \left\{b_{1}, b_{2}\right\}$. Тогда $(M=|Q(N)|)$

$$
M \asymp N^{1 / r}(\log N)^{-b / r}, \quad N \asymp M^{r}(\log M)^{b} .
$$

Предположим для определенности, что $b=b_{1}$. В теоремах 7 и 8 роль "не своих" гиперболических крестов будут играть множества $Q^{\prime}(N) \bigcup_{\mathbf{s} \in \varkappa^{\prime}(N)} \rho(\mathbf{s})$, где $\varkappa^{\prime}(N)=\left\{\mathbf{s}=\left(s_{1}, s_{2}\right): s_{j} \in \mathbb{N}, j=1,2,2^{r s_{1}} s_{1}^{b_{1}} 2^{r s_{2}} s_{2}^{b_{2}^{\prime}} \leqslant N\right\}$, причем $b_{1}<b_{2}^{\prime}<b_{2}$. Из утверждения 1 следует, что

$$
\left|Q^{\prime}(N)\right| \asymp|Q(N)| \asymp N^{1 / r}(\log N)^{-b_{1} / r} .
$$

Как легко убедиться, множество $Q^{\prime}(N)$ шире, чем $Q(N)$.

ТеОрема 7. Пусть $\Omega(\mathbf{t})$ задано равенством (2) с $r>(1 / q-1 / p)_{+}$. Пусть, кроме того, $r<b_{1} \leqslant b_{2}, \quad 1 \leqslant p \leqslant \infty, 1 \leqslant q \leqslant \infty$. Предположим, что при $p=\infty, \quad 1 \leqslant q<\infty$ для $b_{2}$ верны неравенства (12), а при $1 \leqslant q<p<\infty-$ неравенства (13). Пусть, далее, при $1 \leqslant p \leqslant q<\infty$ будет $b_{2}>b_{1}+1 / q_{0}$, $q_{0}=\min \{q, 2\}$, а при $q=\infty$ будет $b_{2}>b_{1}+1$. Тогда

$$
d_{M}^{B}\left(H_{q}^{\Omega}, L_{p}\right) \asymp M^{-r+(1 / q-1 / p)_{+}}(\log M)^{-b_{1}} .
$$

Теорема 8. В условиях теоремы $7,(p, q) \neq(1,1),(\infty, \infty)$, верны порядковые равенства

$$
d_{M}^{\perp}\left(H_{q}^{\Omega}, L_{p}\right) \asymp M^{-r+(1 / q-1 / p)_{+}}(\log M)^{-b_{1}} .
$$

ЗАмЕчАниЕ 4 . K теоремам 7 и 8 также применимо замечание 3 . В теоремах 7 и 8 оптимальными (в смысле порядка) подпространствами являются тригонометрические полиномы со спектром из $Q(N)$ при $q<p$ и из $Q^{\prime}(N)$ при $q \geqslant p$.

ЗАмЕчАнИЕ 5 . Понятно, что в теоремах $3-8$ можно параметры $b_{1}$ и $b_{2}$ поменять местами (т.е., например, в теореме 8 взять $r<b_{2} \leqslant b_{1}$ ) с очевидными изменениями в формулировках. 


\section{§2. Доказательство утверждений и теорем}

ДОКАЗАТЕЛЬСТВО УТВЕРЖДЕНИЯ 1 . Положим $M=N \log ^{-b_{1}} N, m=[\log M]$ (целая часть). Если $k_{1}>(M / 2)^{1 / r}$, то можно показать, что для больших $N$

$$
\left|k_{2}\right|^{r}\left(\log \left|k_{2}\right|\right)_{+}^{b_{2}}<4 r^{b_{1}} .
$$

Пусть

$$
k_{1}\left(r, b_{1}, b_{2}\right)=\max \left\{k \in \mathbb{N}: k^{r}(\log k)_{+}^{b_{2}} \leqslant 4 r^{b_{1}}\right\} .
$$

Если нет натуральных $k$, удовлетворяющих этому неравенству, то положим $k_{1}\left(r, b_{1}, b_{2}\right)=1$. Аналогично пусть

$$
k_{2}\left(r, b_{1}, b_{2}\right)=\max \left\{k \in \mathbb{N}: k^{r}(\log k)_{+}^{b_{1}} \leqslant 4 r^{b_{2}}\right\} .
$$

В случае отсутствия таких $k$ положим $k_{2}\left(r, b_{1}, b_{2}\right)=1$.

Пусть теперь

$$
k\left(r, b_{1}, b_{2}\right)=\max \left\{k_{1}\left(r, b_{1}, b_{2}\right) ; k_{2}\left(r, b_{1}, b_{2}\right)\right\} .
$$

С учетом вьшесказанного будем иметь

$$
\begin{aligned}
|\Gamma(N)| \asymp & \left.\int_{1}^{\left(N \log ^{-b_{1}} N\right)^{1 / r}} d k_{1} \int_{1}^{\left(\frac{N}{k_{1}^{r}\left(\log k_{1}\right)_{+}^{b_{1}}}\left(\log \frac{N}{k_{1}^{r}\left(\log k_{1}\right)_{+}^{b_{1}}}\right)_{+}^{-b_{2}}\right.}\right)^{1 / r} d k_{2} \\
\asymp & k\left(r, b_{1}, b_{2}\right) N^{1 / r}\left(\log ^{-b_{1} / r} N+\log ^{-b_{2} / r} N\right) \\
& +N^{1 / r} \sum_{k=1}^{[m / 2]} \int_{\left(M /\left(2^{k+1}\right)\right)^{1 / r}}^{\left(M /\left(2^{k}\right)\right)^{1 / r}} \frac{1}{k_{1}\left(\log k_{1}\right)^{b_{1} / r}}\left(\log \frac{N}{k_{1}^{r}\left(\log k_{1}\right)^{b_{1}}}\right)_{+}^{-b_{2} / r} d k_{1} \\
& +N^{1 / r} \int_{k\left(r, b_{1}, b_{2}\right)}^{M^{1 /(2 r)}} \frac{1}{k_{1}\left(\log k_{1}\right)_{+}^{b_{1} / r}} \log ^{-b_{2} / r} \frac{N}{k_{1}^{r}\left(\log k_{1}\right)_{+}^{b_{1}}} d k_{1} .
\end{aligned}
$$

Оценим второе слагаемое из правой части (14). Легко видеть, что для $k_{1} \in$ $\left[\left(M /\left(2^{k+1}\right)\right)^{1 / r} ;\left(M /\left(2^{k}\right)\right)^{1 / r}\right]$ будет $\log k_{1} \asymp m-k$. Кроме того, для этих $k_{1}$ при достаточно больших $N$ будет $(k \leqslant[m / 2])$

$$
\log \frac{N}{k_{1}^{r}\left(\log k_{1}\right)^{b_{1}}} \asymp \log \frac{r \cdot 2^{k}}{\left(1-b_{1} \frac{\log \log N}{\log N}-\frac{k}{\log N}\right)^{b_{1}}} \asymp k .
$$

В силу этого получим

$$
\begin{aligned}
N^{1 / r} & \sum_{k=1}^{[m / 2]} \int_{\left(M /\left(2^{k+1}\right)\right)^{1 / r}}^{\left(M /\left(2^{k}\right)\right)^{1 / r}} \frac{1}{k_{1}\left(\log k_{1}\right)^{b_{1} / r}}\left(\log \frac{N}{k_{1}^{r}\left(\log k_{1}\right)^{b_{1}}}\right)_{+}^{-b_{2} / r} d k_{1} \\
\asymp & N^{1 / r} \sum_{k=1}^{[m / 2]}(m-k)^{-b_{1} / r} k^{-b_{2} / r} \int_{\left(M /\left(2^{k+1}\right)\right)^{1 / r}}^{\left(M /\left(2^{k}\right)\right)^{1 / r}} \frac{d k_{1}}{k_{1}} \\
\asymp & N^{1 / r} m^{-b_{1} / r} \sum_{k=1}^{[m / 2]} k^{-b_{2} / r} \asymp N^{1 / r} \begin{cases}m^{-b_{1} / r-b_{2} / r+1}, & b_{2}<r, \\
m^{-b_{1} / r} \log m, & b_{2}=r, \\
m^{-b_{1} / r}, & b_{2}>r .\end{cases}
\end{aligned}
$$


Далее оценим третье слагаемое из правой части (14):

$$
\begin{array}{r}
N^{1 / r} \int_{k\left(r, b_{1}, b_{2}\right)}^{M^{1 /(2 r)}} \frac{1}{k_{1}\left(\log k_{1}\right)_{+}^{b_{1} / r}} \log ^{-b_{2} / r} \frac{N}{k_{1}^{r}\left(\log k_{1}\right)_{+}^{b_{1}}} d k_{1} \\
\asymp N^{1 / r} \log ^{-b_{2} / r} N \int_{k\left(r, b_{1}, b_{2}\right)}^{M^{1 /(2 r)}} \frac{1}{k_{1}\left(\log k_{1}\right)_{+}^{b_{1} / r}} d k_{1} \\
\asymp N^{1 / r} \log ^{-b_{2} / r} N \cdot \begin{cases}(\log M)^{-b_{1} / r+1}, & b_{1}<r, \\
\log \log M, & b_{1}=r, \\
1, & b_{1}>r .\end{cases}
\end{array}
$$

Учитывая, что $\log M \asymp \log N$, собирая воедино все полученные оценки и выбирая наибольшее по порядку слагаемое, получаем утверждение 1.

ДокАЗАТЕЛЬСТво УтВЕРЖДЕнИя 2. Сначала докажем оценку сверху

$$
|\theta(N)| \ll \log N
$$

Прежде всего докажем, что для любого $s_{1} \in \mathbb{N}$ такого, что $\mathbf{s}=\left(s_{1}, s_{2}\right) \in \theta(N)$, существует лишь конечное число $n_{2} \in \mathbb{N}$ такое, что $\left(s_{1}, s_{2}+n_{2}\right) \in \theta(N)$.

Действительно, если $\mathbf{s}=\left(s_{1}, s_{2}\right) \in \theta(N)$ и $\left(s_{1}, s_{2}+n_{2}\right) \in \theta(N)$, то в силу (8)

$$
1 \leqslant \frac{\Omega\left(2^{-\mathbf{s}}\right)}{\Omega\left(2^{-s_{1}} ; 2^{-\left(s_{2}+n_{2}\right)}\right)} \leqslant 2^{l}
$$

Из (2) следует, что функция

$$
y(\mathbf{t})=\frac{\Omega(\mathbf{t})}{\left(t_{1} t_{2}\right)^{r / 2}}
$$

почти возрастает, т.е. для векторов

$$
\mathbf{t}^{(1)}=\left(t_{1}^{(1)} ; t_{2}^{(1)}\right), \quad \mathbf{t}^{(2)}=\left(t_{1}^{(2)} ; t_{2}^{(2)}\right), \quad t_{j}^{(1)} \leqslant t_{j}^{(2)}, \quad j=1,2,
$$

верно неравенство

$$
y\left(\mathbf{t}^{(1)}\right) \leqslant C_{1} y\left(\mathbf{t}^{(2)}\right),
$$

где число $C_{1}>0$ не зависит от $\mathbf{t}$ (можно считать $C_{1}>1$ ). Следовательно,

$$
\frac{\Omega\left(2^{-s_{1}} ; 2^{-\left(s_{2}+n_{2}\right)}\right)}{2^{-r\left(s_{2}+n_{2}\right) / 2}} \leqslant C_{1} \frac{\Omega\left(2^{-s}\right)}{2^{-r s_{2} / 2}} .
$$

Учитывая это неравенство, а также $(15)$, для $\mathbf{s}$ и $\left(s_{1}, s_{2}+n_{2}\right)$ из $\theta(N)$ получим $2^{r n_{2} / 2} \leqslant C_{1} \cdot 2^{l}$, т.e.

$$
n_{2} \leqslant \frac{2 l+2 \log C_{1}}{r} \equiv m_{2} .
$$

Итак, мы доказали ограниченность $n_{2}$.

Далее, учитывая почти возрастание функции (16), мы получаем

$$
\Omega(\mathbf{t})=\frac{\Omega(\mathbf{t})}{\left(t_{1} t_{2}\right)^{r / 2}}\left(t_{1} t_{2}\right)^{r / 2} \leqslant C_{1} \Omega(1,1)\left(t_{1} t_{2}\right)^{r / 2},
$$


T.e.

$$
\Omega(\mathbf{t}) \leqslant C_{2}\left(t_{1} t_{2}\right)^{r / 2}
$$

Рассмотрим функцию

$$
z(\mathbf{t})=\frac{\Omega(\mathbf{t})}{\left(t_{1} t_{2}\right)^{l}} .
$$

Так как $\Omega(\mathbf{t})$ определено в $(2)$ и $r<l$, то функция $z(\mathbf{t})$ почти убывает, т.е. для векторов $\mathbf{t}^{(1)}$ и $\mathbf{t}^{(2)}$ из (17) верно неравенство

$$
z\left(\mathbf{t}^{(1)}\right) \geqslant C_{3} z\left(\mathbf{t}^{(2)}\right)
$$

где $C_{3}>0$ не зависит от $\mathbf{t}$.

Аналогично неравенству (19) получаем неравенство

$$
\Omega(\mathbf{t}) \geqslant C_{4}\left(t_{1} t_{2}\right)^{l},
$$

причем мы можем считать $0<C_{4}<C_{2}$. Из (8), (19), (20) следует, что

$$
\frac{1}{l} \log \left(C_{4} N\right)<s_{1}+s_{2} \leqslant \frac{2}{r} \log \left(C_{2} 2^{l} N\right) .
$$

Пусть для натурального $n$

$$
\theta_{n}=\left\{\mathbf{s}=\left(s_{1}, s_{2}\right): s_{j} \in \mathbb{N}, \quad j=1,2 ; \quad s_{1}+s_{2}=n\right\} .
$$

Очевидно, $\left|\theta_{n}\right|=n$. В силу замечания 2 и $(21)$ множество $\theta(N)$ можно взаимно однозначно отобразить на некоторое подмножество множества

$$
A_{N}=\bigcup_{n=m_{1}}^{m_{1}+m_{2}} \theta_{n},
$$

где $m_{1}=\left[(2 / r) \log \left(C_{2} 2^{l} N\right)\right]+1, m_{2}$ определено в (18). Здесь квадратные скобки обозначают целую часть числа. Из (22) мы получаем оценку сверху.

Далее, для $\mathbf{s} \in \theta(N)$ верно неравенство

$$
\log N<s_{1} r+b_{1} \log s_{1}+s_{2} r+b_{2} \log s_{2} \leqslant l+\log N,
$$

из которого следует, что $1 \ll s_{1} \ll \log N$. Учитывая это неравенство и замечание 2 , мы получим, что $|\theta(N)| \gg \log N$. Следовательно, $|\theta(N)| \asymp \log N$. Утверждение 2 доказано.

Из того факта, что $\Omega(\mathbf{t})$, заданная равенством (2), удовлетворяет условию (S), можно вывести следующие леммы.

ЛЕмма 1. Для функции $\Omega(\mathbf{t})$, определенной равенством (2), при $0<p<\infty$ верно неравенство

$$
\sum_{\mathbf{s} \in \varkappa^{\perp}(N)}\left(\Omega\left(2^{-\mathbf{s}}\right)\right)^{p} \ll \sum_{\mathbf{s} \in \theta(N)}\left(\Omega\left(2^{-\mathbf{s}}\right)\right)^{p}
$$


ЛЕмма 2. Пусть $\Omega(\mathbf{t})$ определено формулой (2), причем $r>\beta>0$. Тогда при $0<p<\infty$

$$
\sum_{\mathbf{s} \in \varkappa^{\perp}(N)}\left(\Omega\left(2^{-\mathbf{s}}\right) \cdot 2^{\|\mathbf{s}\|_{1} \beta}\right)^{p} \ll \sum_{\mathbf{s} \in \theta(N)}\left(\Omega\left(2^{-\mathbf{s}}\right) \cdot 2^{\|\mathbf{s}\|_{1} \beta}\right)^{p} .
$$

ДоКАЗАТЕЛЬСТВо ТЕОРЕм 1 и 2. Сначала докажем оценки сверху. В силу неравенства (1) их достаточно доказать для $d_{M}^{\perp}\left(H_{q}^{\Omega}, L_{p}\right)$ в случаях, охваченных теоремой 2 . При $(p, q)=(1,1),(\infty, \infty)$ оценки сверху будут верны лишь для $d_{M}^{B}\left(H_{q}^{\Omega}, L_{p}\right)$.

1) $1 \leqslant p \leqslant q<\infty, q>1$. Используя возрастание $L_{p}$-нормы по параметру $p$, теорему А и следствие из теоремы Литтлвуда-Пэли, получим

$$
E_{Q(N)}\left(H_{q}^{\Omega}\right)_{p} \leqslant E_{Q(N)}\left(H_{q}^{\Omega}\right)_{q} \ll\left(\sum_{\mathbf{s} \in \varkappa^{\perp}(N)}\left(\Omega\left(2^{-\mathbf{s}}\right)\right)^{q_{0}}\right)^{1 / q_{0}}
$$

Здесь через $E_{Q(N)}\left(H_{q}^{\Omega}\right)_{p}$ обозначена величина

$$
E_{Q(N)}\left(H_{q}^{\Omega}\right)_{p}=\sup _{f \in H_{q}^{\Omega}} E_{Q(N)}(f)_{p},
$$

где $E_{Q(N)}(f)_{p}$ - наилучшее приближение функции $f(\mathbf{x})$ тригонометрическими полиномами с гармониками из множества $Q(N)$ в норме $L_{p}$.

Далее используем лемму $1,(9)$ и (10) и приходим к оценке

$$
E_{Q(N)}\left(H_{q}^{\Omega}\right)_{p} \ll \frac{1}{N}|\theta(N)|^{1 / q_{0}} \asymp M^{-r}(\log M)^{-b_{1}-b_{2}+r+1 / q_{0}} .
$$

2) $1 \leqslant p<q=\infty$. В этом случае имеем

$E_{Q(N)}\left(H_{\infty}^{\Omega}\right)_{p} \ll E_{Q(N)}\left(H_{p+1}^{\Omega}\right)_{p+1} \ll \frac{1}{N}(\log N)^{1 / 2} \asymp M^{-r}(\log M)^{-b_{1}-b_{2}+r+1 / 2}$.

Здесь мы использовали то, что $p+1 \geqslant 2$.

3) $1 \leqslant q<p<\infty, \beta=1 / q-1 / p$. По лемме А и лемме 2 имеем

$$
E_{Q(N)}\left(H_{q}^{\Omega}\right)_{p} \ll\left(\sum_{\mathbf{s} \in \varkappa \perp(N)}\left(\Omega\left(2^{-\mathbf{s}}\right) \cdot 2^{\|\mathbf{s}\|_{1} \beta}\right)^{p}\right)^{1 / p} \ll \frac{1}{N}\left(\sum_{\mathbf{s} \in \theta(N)} 2^{\|\mathbf{s}\|_{1}(p / q-1)}\right)^{1 / p} .
$$

Оценим сумму, стоящую в скобках. Для этого разобьем ее на три суммы, соответствуюшие множествам (см. (21))

$$
\begin{aligned}
& \theta_{1}(N)=\left\{\mathbf{s} \in \theta(N): s_{j} \geqslant \frac{1}{4 l} \log \left(C_{4} N\right), \quad j=1,2\right\}, \\
& \theta_{2}(N)=\left\{\mathbf{s} \in \theta(N): s_{1}<\frac{1}{4 l} \log \left(C_{4} N\right), \quad s_{2}>\frac{3}{4 l} \log \left(C_{4} N\right)\right\}, \\
& \theta_{3}(N)=\left\{\mathbf{s} \in \theta(N): s_{2}<\frac{1}{4 l} \log \left(C_{4} N\right), \quad s_{1}>\frac{3}{4 l} \log \left(C_{4} N\right)\right\} .
\end{aligned}
$$


Оценим каждую сумму отдельно.

Рассмотрим первую сумму, соответствующую множеству $\theta_{1}(N)$. Из соотношения $(21)$ с учетом замечания 2 следует, что множество

$$
\begin{gathered}
\theta_{N}^{\prime}=\left\{\mathbf{s}=\left(s_{1}, s_{2}\right): \quad s_{j} \in \mathbb{N}, \quad j=1,2, \quad s_{1}+s_{2}=\left[\frac{1}{l} \log \left(C_{4} N\right)\right],\right. \\
\left.s_{j} \geqslant \frac{1}{4 l} \log \left(C_{4} N\right), \quad j=1,2\right\}
\end{gathered}
$$

можно взаимно однозначно отобразить на некоторое подмножество множества $\theta_{1}(N)$, откуда следует, что $\left|\theta_{1}(N)\right| \asymp \log N$. Для $\mathbf{s}=\left(s_{1}, s_{2}\right) \in \theta_{1}(N)$ будет $s_{j} \asymp \log N$ и $2^{r s_{1}} s_{1}^{b_{1}} 2^{r s_{2}} s_{2}^{b_{2}} \asymp N$, откуда в свою очередь следует, что

$$
2^{\|\mathbf{s}\|_{1}} \asymp N^{1 / r}(\log N)^{-b_{1} / r-b_{2} / r} .
$$

Далее имеем

$$
\sum_{\mathbf{s} \in \theta_{1}(N)} 2^{\|\mathbf{s}\|_{1}(p / q-1)} \asymp\left(N^{1 / r}(\log N)^{-b_{1} / r-b_{2} / r}\right)^{p / q-1} \log N .
$$

Возьмем вторую сумму, соответствуюшую множеству $\theta_{2}(N)$.

Пусть

$$
n_{1}=\left[\log \left(\max \left\{s_{1} \in \mathbb{N}:\left(s_{1}, s_{2}\right) \in \theta(N)\right\}\right)\right] \asymp \log \log N .
$$

Тогда

$$
\begin{aligned}
& \sum_{\mathbf{s} \in \theta_{2}(N)} 2^{\|\mathbf{s}\|_{1}(p / q-1)} \asymp \sum_{t=1}^{n_{1}} \sum_{s_{1}=2^{t-1}}^{2^{t}-1} 2^{\|\mathbf{s}\|_{1}(p / q-1)} \\
& \asymp \sum_{t=1}^{n_{1}} \sum_{s_{1}=2^{t-1}}^{2^{t}-1}\left(N^{1 / r}(\log N)^{-b_{2} / r} 2^{-t b_{1} / r}\right)^{p / q-1} \\
& \asymp\left(N^{1 / r}(\log N)^{-b_{2} / r}\right)^{p / q-1} \sum_{t=1}^{n_{1}} 2^{t\left(1-b_{1}(p / q-1) / r\right)} \\
& \asymp\left(N^{1 / r}(\log N)^{-b_{2} / r}\right)^{p / q-1} \cdot \begin{cases}(\log N)^{1-b_{1}(p / q-1) / r}, & \frac{b_{1}}{r}\left(\frac{p}{q}-1\right)<1, \\
\log \log N, & \frac{b_{1}}{r}\left(\frac{p}{q}-1\right)=1, \\
1, & \frac{b_{1}}{r}\left(\frac{p}{q}-1\right)>1 .\end{cases}
\end{aligned}
$$

Аналогично для третьей суммы, соответствующей $\theta_{3}(N)$, будет справедлива оценка

$$
\begin{aligned}
& \sum_{\mathbf{s} \in \theta_{3}(N)} 2^{\|\mathbf{s}\|_{1}(p / q-1)} \asymp \\
& \asymp\left(N^{1 / r}(\log N)^{-b_{1} / r}\right)^{p / q-1} \cdot \begin{cases}(\log N)^{1-b_{2}(p / q-1) / r}, & \frac{b_{2}}{r}\left(\frac{p}{q}-1\right)<1, \\
\log \log N, & \frac{b_{2}}{r}\left(\frac{p}{q}-1\right)=1, \\
1, & \frac{b_{2}}{r}\left(\frac{p}{q}-1\right)>1 .\end{cases}
\end{aligned}
$$


В итоге с учетом $b_{j}<\frac{r}{p(1 / q-1 / p)}$ получаем оценку сверху

$$
\begin{aligned}
E_{Q(N)}\left(H_{q}^{\Omega}\right)_{p} & \ll \frac{1}{N}\left(N^{1 / r}(\log N)^{-b_{1} / r-b_{2} / r}\right)^{1 / q-1 / p}(\log N)^{1 / p} \\
& \asymp M^{-r+1 / q-1 / p}(\log M)^{-b_{1}-b_{2}+r+2 / p-1 / q} .
\end{aligned}
$$

4) $p=\infty, 1 \leqslant q<\infty, \beta=1 / q$. Легко видеть, что оператор взятия двоичной пачки $\delta_{\mathbf{s}}(f ; \mathbf{x})$ как оператор из $L_{q}$ в $L_{\infty}$ имеет норму, по порядку не превосходяшую $2^{\|s\|_{1} / q}$. В качестве ортопроектора рассмотрим

$$
S_{Q(N)}(f ; \mathbf{x})=\sum_{\mathbf{s} \in \varkappa(N)} \delta_{\mathbf{s}}(f ; \mathbf{x}) .
$$

С учетом теоремы А и леммы 2 для $f \in H_{q}^{\Omega}$ получим

$$
\begin{aligned}
& \left\|f(\mathbf{x})-S_{Q(N)}(f ; \mathbf{x})\right\|_{\infty} \leqslant \sum_{\mathbf{s} \in \varkappa^{\perp}(N)}\left\|\delta_{\mathbf{s}}(f ; \mathbf{x})\right\|_{\infty} \\
& \quad=\sum_{\mathbf{s} \in \varkappa^{\perp}(N)}\left\|\delta_{\mathbf{s}}\left(\sum_{\left\|\mathbf{s}^{\prime}-\mathbf{s}\right\|_{\infty} \leqslant 1} A_{\mathbf{s}^{\prime}}(f ; \mathbf{x})\right)\right\|_{\infty} \ll \sum_{\substack{\mathbf{s} \in \varkappa^{\perp}(N) \\
\left\|\mathbf{s}^{\prime}-\mathbf{s}\right\|_{\infty} \leqslant 1}}\left\|A_{\mathbf{s}^{\prime}}(f ; \mathbf{x})\right\|_{q} \cdot 2^{\|\mathbf{s}\|_{1} / q} \\
& \quad \ll \sum_{\mathbf{s} \in \varkappa^{\perp}(N)} \Omega\left(2^{-\mathbf{s}}\right) \cdot 2^{\|\mathbf{s}\|_{1} / q} \ll \frac{1}{N} \sum_{\mathbf{s} \in \theta(N)} 2^{\|\mathbf{s}\|_{1} / q} .
\end{aligned}
$$

Далее покажем, что

$$
\sum_{\mathbf{s} \in \theta(N)} 2^{\|\mathbf{s}\|_{1}} \asymp|Q(N)| .
$$

Действительно, пусть $u_{1}=\max \left\{s_{1} \in \mathbb{N}:\left(s_{1}, s_{2}\right) \in \varkappa(N)\right\} ;$ для данного $s_{1} \in \mathbb{N}$ положим $u_{2}\left(s_{1}\right)=\max \left\{s_{2} \in \mathbb{N}:\left(s_{1}, s_{2}\right) \in \varkappa(N)\right\}$. Тогда

$$
|Q(N)|=\sum_{s_{1}=1}^{u_{1}} \sum_{s_{2}=1}^{u_{2}\left(s_{1}\right)}|\rho(\mathbf{s})| \asymp \sum_{s_{1}=1}^{u_{1}} 2^{s_{1}+u_{2}\left(s_{1}\right)} \asymp \sum_{\mathbf{s} \in \theta(N)} 2^{\|\mathbf{s}\|_{1}}
$$

(см. замечание 2). Соотношение (25) доказано.

Применив неравенство Гёльдера и $(25)\left(1 / q+1 / q^{\prime}=1\right)$, продолжаем оценку cверxy:

$$
\begin{aligned}
\| f(\mathbf{x}) & -S_{Q(N)}(f ; \mathbf{x}) \|_{\infty} \ll \frac{1}{N}\left(\sum_{\mathbf{s} \in \theta(N)} 1\right)^{1 / q^{\prime}}\left(\sum_{\mathbf{s} \in \theta(N)} 2^{\|\mathbf{s}\|_{1}}\right)^{1 / q} \\
\ll & \frac{1}{N}(\log N)^{1 / q^{\prime}}|Q(N)|^{1 / q} \asymp M^{-r+1 / q}(\log M)^{-b_{1}-b_{2}+r+1-1 / q} .
\end{aligned}
$$

5) $(p, q)=(1,1),(\infty, \infty)$. Рассмотрим оператор

$$
V_{Q(N)}(f ; \mathbf{x})=\sum_{\mathbf{s} \in \varkappa(N)} A_{\mathbf{s}}(f ; \mathbf{x}),
$$


где $A_{\mathbf{s}}(f ; \mathbf{x})$ определено в $(6)$. Нетрудно убедиться в том, что этот оператор принадлежит множеству $\mathscr{L}_{M}(1)_{p}$, где $M=|Q(N)|$. Тогда для $f(\mathbf{x}) \in H_{q}^{\Omega}$ имеем $(p=1, \infty)$

$$
\left\|f(\mathbf{x})-V_{Q(N)}(f ; \mathbf{x})\right\|_{p} \leqslant \sum_{\mathbf{s} \in \varkappa^{\perp}(N)}\left\|A_{\mathbf{s}}(f ; \mathbf{x})\right\|_{p} \ll \sum_{\mathbf{s} \in \varkappa^{\perp}(N)} \Omega\left(2^{-\mathbf{s}}\right)
$$

в силу теоремы А. Далее по лемме 1 и (9) получим

$$
E_{Q(N)}\left(H_{p}^{\Omega}\right)_{p} \ll \frac{1}{N} \log N \asymp M^{-r}(\log M)^{-b_{1}-b_{2}+r+1} .
$$

Перейдем к доказательству оценок снизу. В силу неравенства (1) их достаточно доказать для $d_{M}^{B}\left(H_{q}^{\Omega}, L_{p}\right)$. Будем придерживаться схемы, использованной В.Н. Темляковым в [3]. Сформулируем лемму, доказанную в [3].

ЛЕмма 3. Рассмотрим линейный оператор, заданный следующим образом:

$$
A e^{i(\mathbf{k}, \mathbf{x})}=\sum_{m=1}^{L} a_{m}^{\mathbf{k}} \psi_{m}(\mathbf{x})
$$

где $\left\{\psi_{m}(\mathbf{x})\right\}_{m=1}^{L}-$ набор функиий, для которых $\left\|\psi_{m}(\mathbf{x})\right\|_{2} \leqslant 1, \quad m=1, \ldots, L$. Тогда для любого тригонометрического полинома $t(\mathbf{x})$

$$
\min _{\mathbf{y}=\mathbf{x}} \operatorname{Re} A t(\mathbf{x}-\mathbf{y}) \leqslant\left(L \sum_{m=1}^{L} \sum_{\mathbf{k}}\left|a_{m}^{\mathbf{k}} \hat{t}(\mathbf{k})\right|^{2}\right)^{1 / 2}
$$

1) $p=\infty, 1 \leqslant q \leqslant \infty$. Рассмотрим функцию

$$
\Phi(\mathbf{x})=\sum_{\mathbf{s} \in \theta_{1}(N)} K_{\mathbf{s}}(\mathbf{x})
$$

где $\theta_{1}(N)$ определено в $(23)$,

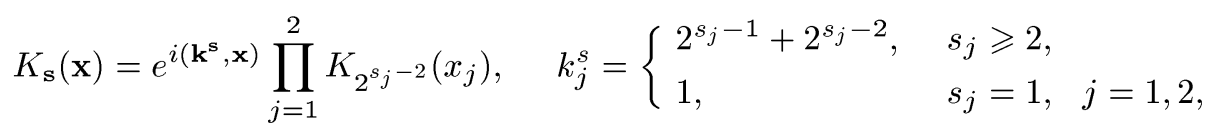

где $K_{m}(\tau)$ - ядро Фейера.

Эта функция аналогична функции из примера 2 работы [3].

Как было отмечено выше,

$$
\left|\theta_{1}(N)\right| \asymp \log N .
$$

Предположим, что оператор $G$ принадлежит $\mathscr{L}_{M}(B)_{\infty}$. Покажем, что существует вектор $\mathbf{y}^{*}=\left(y_{1}^{*}, y_{2}^{*}\right)$ такой, что

$$
\left\|\Phi\left(\mathbf{x}-\mathbf{y}^{*}\right)-G \Phi\left(\mathbf{x}-\mathbf{y}^{*}\right)\right\|_{\infty} \gg M
$$


Очевидно,

$$
\|\Phi(\mathbf{x}-\mathbf{y})-G \Phi(\mathbf{x}-\mathbf{y})\|_{\infty} \geqslant \Phi(\mathbf{0})-\min _{\mathbf{y}=\mathbf{x}} \operatorname{Re} G \Phi(\mathbf{x}-\mathbf{y})
$$

По лемме 3

$$
\min _{\mathbf{y}=\mathbf{x}} \operatorname{Re} G \Phi(\mathbf{x}-\mathbf{y}) \leqslant M^{1 / 2} B\left(\sum_{\mathbf{k}}|\widehat{\Phi}(\mathbf{k})|^{2}\right)^{1 / 2} \ll M^{1 / 2} B\left|\Theta_{1}(N)\right|^{1 / 2} .
$$

Здесь через $\left|\Theta_{1}(N)\right|$ обозначено количество элементов множества

$$
\Theta_{1}(N)=\bigcup_{\mathbf{s} \in \theta_{1}(N)} \rho(\mathbf{s})
$$

Учитывая $(24),(28)$, а также то, что $|\rho(\mathbf{s})|=2^{\|\mathbf{s}\|_{1}}$, получим

$$
\left|\Theta_{1}(N)\right| \asymp N^{1 / r}(\log N)^{-b_{1} / r-b_{2} / r+1} .
$$

С другой стороны,

$$
\Phi(\mathbf{0}) \asymp N^{1 / r}(\log N)^{-b_{1} / r-b_{2} / r+1} \asymp\left|\Theta_{1}(N)\right| .
$$

С учетом (30) и (32) можно подобрать такое $N$, что $\left|\Theta_{1}(N)\right| \asymp M$ и правая часть в (33) будет по крайней мере вдвое больше правой части (30). Для этого $N$ при некотором $\mathbf{y}^{*}$ имеем

$$
\left\|\Phi\left(\mathbf{x}-\mathbf{y}^{*}\right)-G \Phi\left(\mathbf{x}-\mathbf{y}^{*}\right)\right\|_{\infty} \gg M
$$

Таким образом оценка (29) доказана.

Далее рассмотрим функцию

$$
\Phi_{1}(\mathbf{x})=C \frac{1}{N}\left(N^{1 / r}(\log N)^{-b_{1} / r-b_{2} / r}\right)^{1 / q-1} \Phi(\mathbf{x})
$$

где $\Phi(\mathbf{x})$ взято из (27). Так как

$$
\left\|K_{\mathbf{s}}(\mathbf{x})\right\|_{q} \asymp 2^{\|\mathbf{s}\|_{1}(1-1 / q)}, \quad 1 \leqslant q \leqslant \infty,
$$

то функция (34) по теореме А принадлежит $H_{q}^{\Omega}$ при некотором $C>0$. С учетом (29) будет

$$
\left\|\Phi_{1}\left(\mathbf{x}-\mathbf{y}^{*}\right)-G \Phi_{1}\left(\mathbf{x}-\mathbf{y}^{*}\right)\right\|_{\infty} \gg M^{-r+1 / q}(\log M)^{-b_{1}-b_{2}+r+1-1 / q} .
$$

2) $1 \leqslant q<p<\infty$. Рассмотрим оператор

$$
A=\left(S_{Q\left(2^{l} N\right)}-S_{Q(N)}\right) G
$$

где $S_{Q(N)}$ - оператор взятия частичной суммы Фурье, соответствующей множеству $Q(N), G \in \mathscr{L}_{M}(B)_{p}$. 
Для тригонометрического полинома $t(\mathbf{x})$ со спектром из $\Theta_{1}(N)$ (см. (31)) будет

$$
t(\mathbf{x})=t(\mathbf{x}) *\left(\sum_{\mathbf{k} \in \Theta_{1}(N)} e^{i(\mathbf{k}, \mathbf{x})}\right)
$$

Тогда

$$
\|t(\mathbf{x})\|_{\infty} \leqslant\|t(\mathbf{x})\|_{p}\left\|_{\mathbf{k} \in \Theta_{1}(N)} e^{i(\mathbf{k}, \mathbf{x})}\right\|_{p^{\prime}}
$$

Здесь $p^{\prime}=p /(p-1)$. Оценим сверху $\left\|\sum_{\mathbf{k} \in \Theta_{1}(N)} e^{i(\mathbf{k}, \mathbf{x})}\right\|_{p^{\prime}}$. Возьмем $\gamma: 1<\gamma<p^{\prime}$. Тогда с учетом леммы A, (24) и оценки

$$
\left\|\sum_{\mathbf{k} \in \rho(\mathbf{s})} e^{i(\mathbf{k}, \mathbf{x})}\right\|_{\gamma} \asymp 2^{\|\mathbf{s}\|_{1}(1-1 / \gamma)}
$$

мы получим

$$
\begin{aligned}
\left\|\sum_{\mathbf{k} \in \Theta_{1}(N)} e^{i(\mathbf{k}, \mathbf{x})}\right\|_{p^{\prime}}^{p^{\prime}} \ll \sum_{\mathbf{s} \in \theta_{1}(N)}\left\|\sum_{\mathbf{k} \in \rho(\mathbf{s})} e^{i(\mathbf{k}, \mathbf{x})}\right\|_{\gamma}^{p^{\prime}} \cdot 2^{\|\mathbf{s}\|_{1}\left(p^{\prime} / \gamma-1\right)} \\
\quad \ll \sum_{\mathbf{s} \in \theta_{1}(N)} 2^{\|\mathbf{s}\|_{1}\left(p^{\prime}-1\right)} \ll\left(N^{1 / r}(\log N)^{-b_{1} / r-b_{2} / r}\right)^{p^{\prime}-1} \log N .
\end{aligned}
$$

В итоге получаем

$$
\|t(\mathbf{x})\|_{\infty} \ll\|t(\mathbf{x})\|_{p}\left(N^{1 / r}(\log N)^{-b_{1} / r-b_{2} / r}\right)^{1 / p}(\log N)^{1-1 / p} .
$$

Рассмотрим функцию $\Phi_{1}(\mathbf{x})$ из (34). В силу (29) существует $\mathbf{y}^{*}$ такой, что

$$
\begin{aligned}
& M^{-r+1 / q}(\log M)^{-b_{1}-b_{2}+r+1-1 / q} \ll\left\|\Phi_{1}\left(\mathbf{x}-\mathbf{y}^{*}\right)-A \Phi_{1}\left(\mathbf{x}-\mathbf{y}^{*}\right)\right\|_{\infty} \\
& \quad \ll\left\|\Phi_{1}\left(\mathbf{x}-\mathbf{y}^{*}\right)-G \Phi_{1}\left(\mathbf{x}-\mathbf{y}^{*}\right)\right\|_{p}\left(N^{1 / r}(\log N)^{-b_{1} / r-b_{2} / r}\right)^{1 / p}(\log N)^{1-1 / p},
\end{aligned}
$$

откуда получаем требуемую оценку снизу.

3) $1<q=p \leqslant 2$. Взяв функцию $\Phi_{1}(\mathbf{x})$ из (34) с $q=p$, аналогично предыдущему случаю получаем оценку снизу.

4) $1 \leqslant p \leqslant q, q \geqslant 2, p<\infty$. В этом пункте рассмотрим функцию, аналогичную функции из примера 6 работы [3].

Пусть $G \in \mathscr{L}_{M}(B)_{1}$. Тогда найдутся $N, \theta_{4}(N), \theta_{4}(N) \subset \theta(N)$, такие, что

$$
\left|\theta_{4}(N)\right| \geqslant \frac{1}{2}|\theta(N)|, \quad|\Theta(N)| \asymp M,
$$

где

$$
\Theta(N)=\bigcup_{\mathbf{s} \in \theta(N)} \rho(\mathbf{s})
$$


и в каждом $\rho(\mathbf{s}), \mathbf{s} \in \theta_{4}(N)$, найдутся векторы $\mathbf{k}^{\mathbf{s}}$, при которых для функции

$$
g(\mathbf{x})=\sum_{\mathbf{s} \in \theta_{4}(N)} e^{i\left(\mathbf{k}^{\mathbf{s}}, \mathbf{x}\right)}
$$

найдется $\mathbf{y}^{*}=\left(\mathbf{y}_{1}^{*}, \mathbf{y}_{2}^{*}\right)$ такой, что

$$
\left\|g\left(\mathbf{x}+\mathbf{y}^{*}\right)-G g\left(\mathbf{x}+\mathbf{y}^{*}\right)\right\|_{1} \gg(\log M)^{1 / 2} .
$$

Доказательство этого факта аналогично доказательству примера 6 из [3].

Рассмотрим функцию $g_{1}(\mathbf{x})=C \cdot(1 / N) g(\mathbf{x})$, где $g(\mathbf{x})$ взято из $(36)$. По теореме А $g_{1}(\mathbf{x}) \in H_{q}^{\Omega}$ при некотором $C>0$. В силу $(37)$ получим

$$
\begin{gathered}
\left\|g_{1}\left(\mathbf{x}+\mathbf{y}^{*}\right)-G g\left(\mathbf{x}+\mathbf{y}^{*}\right)\right\|_{p} \geqslant\left\|g_{1}\left(\mathbf{x}+\mathbf{y}^{*}\right)-G g_{1}\left(\mathbf{x}+\mathbf{y}^{*}\right)\right\|_{1} \\
\gg \frac{1}{N}(\log M)^{1 / 2} \asymp M^{-r}(\log M)^{-b_{1}-b_{2}+r+1 / 2} .
\end{gathered}
$$

5) $1 \leqslant p \leqslant q \leqslant 2$. Функция, дающая оценку снизу в этом случае, аналогична функции из примера 7 работы [3].

Пусть $N$ достаточно велико. Возьмем множество $\theta_{1}(N)$, определенное равенством (23). Положим $v=\left[\left|\theta_{1}(N)\right|^{1 / 2}\right]$ и разобьем куб $\pi_{2}=[-\pi ; \pi]^{2}$ на $v^{2}$ кубов с ребром $2 \pi / v$. Установим взаимно однозначное соответствие между множеством $\theta_{1}^{\prime}(N) \subset \theta_{1}(N),\left|\theta_{1}^{\prime}(N)\right|=v^{2}$, и получившимся множеством кубов.

Для $\mathbf{s} \in \theta_{1}^{\prime}(N)$ через $\mathbf{x}^{\mathbf{s}}$ обозначим центр соответствующего куба. Положим

$$
u=2^{\left[(1 / 2) \log \left|\theta_{1}(N)\right|\right]} \asymp(\log N)^{1 / 2} .
$$

Пусть $G \in \mathscr{L}_{M}(B)_{1}$. Найдутся $N$ и множество $\theta_{5}(N) \subset \theta_{1}^{\prime}(N)$ такие, что $|\Theta(N)| \asymp M$ (см. (35)), $\left|\theta_{5}(N)\right| \geqslant(1 / 2)\left|\theta_{1}^{\prime}(N)\right|$ и в каждом $\rho(\mathbf{s}), \mathbf{s} \in \theta_{5}(N)$, кубы $\Delta_{\mathbf{s}} \subset \rho(\mathbf{s})$ с центрами $\mathbf{k}^{\mathbf{s}}$ и ребром $2 u$ такие, что для функции $\left(K_{m}(\tau)-\right.$ ядра Фейера)

$$
h(\mathbf{x})=\sum_{\mathbf{s} \in \theta_{5}(N)} e^{i\left(\mathbf{k}^{\mathbf{s}}, \mathbf{x}\right)} \prod_{j=1}^{2} K_{u}\left(x_{j}-x_{j}^{s}\right)
$$

найдется $\mathbf{y}^{*}$ такое, что

$$
\left\|h\left(\mathbf{x}+\mathbf{y}^{*}\right)-G h\left(\mathbf{x}+\mathbf{y}^{*}\right)\right\|_{1} \gg \log M .
$$

Отметим, что при достаточно большом $N$ условие (38) обеспечивает включение $\Delta_{\mathbf{s}} \subset \rho(\mathbf{s})$.

Доказательство (40) полностью аналогично доказательству примера 7 из [2].

Рассмотрим функцию

$$
h_{1}(\mathbf{x})=C \frac{(\log N)^{1 / q-1}}{N} h(\mathbf{x})
$$

с функцией $h(\mathbf{x})$ из $(39)$. Функция $h_{1}(\mathbf{x})$ принадлежит $H_{q}^{\Omega}$ при некотором $C>0$. Из (40) следует, что

$$
\left\|h_{1}\left(\mathbf{x}+\mathbf{y}^{*}\right)-G h_{1}\left(\mathbf{x}+\mathbf{y}^{*}\right)\right\|_{p} \gg \frac{(\log N)^{1 / q}}{N} \asymp M^{-r}(\log M)^{-b_{1}-b_{2}+r+1 / q} .
$$

Теоремы 1 и 2 доказаны.

В других случаях значений параметров $b_{j}, j=1,2$, доказательства сходны с доказательством теорем 1 и 2. Укажем лиш некоторые основные отличия в доказательствах. 
ДоКАЗАТЕЛЬСТВО ТЕОРЕм 5,6 . Оценки сверху в случае $q<p$ доказываются аналогично оценкам сверху в теоремах 1 и 2 . Докажем оценки сверху в случае $1<p \leqslant q<\infty$. В качестве приближаюшего подпространства возьмем множество тригонометрических полиномов с гармониками из множеств $Q^{\prime}(N)$.

По следствию из теоремы Литтлвуда-Пэли

$$
\begin{aligned}
E_{Q^{\prime}(N)}\left(H_{q}^{\Omega}\right)_{q} & \ll\left(\sum_{\mathbf{s} \in\left(\varkappa^{\prime}\right)^{\perp}(N)}\left(\Omega\left(2^{-\mathbf{s}}\right)\right)^{q_{0}}\right)^{1 / q_{0}} \\
& \leqslant\left(\sum_{\mathbf{s} \in \varkappa^{\perp}\left(N(\log N)^{\left.b_{2}-b_{2}^{\prime}\right)}\right.}\left(\Omega\left(2^{-\mathbf{s}}\right)\right)^{1 / q_{0}}\right)+\left(\sum_{\mathbf{s} \in A(N)}\left(\Omega\left(2^{-\mathbf{s}}\right)\right)^{q_{0}}\right)^{1 / q_{0}}
\end{aligned}
$$

где $A(N)=\left(\varkappa^{\prime}\right)^{\perp}(N) \backslash \varkappa^{\perp}\left(N(\log N)^{b_{2}-b_{2}^{\prime}}\right)$.

Первая сумма из правой части (41) оценивается по лемме 1 :

$$
\sigma_{1} \ll \frac{1}{N(\log N)^{b_{2}-b_{2}^{\prime}}}(\log N)^{1 / q_{0}} .
$$

Оценим вторую сумму $\sigma_{2}$ из правой части (41). Для векторов $\mathbf{s}=\left(s_{1}, s_{2}\right)$ из $\sigma_{2}$ будет

$$
2^{r s_{1}} s_{1}^{b_{1}} 2^{r s_{2}} s_{2}^{b_{2}^{\prime}}>N, \quad 2^{r s_{1}} s_{1}^{b_{1}} 2^{r s_{2}} s_{2}^{b_{2}} \leqslant N(\log N)^{b_{2}-b_{2}^{\prime}} .
$$

Если $s_{2} \geqslant \log N /(6 r)$, то из (42) и того, что $\mathbf{s}=\left(s_{1}, s_{2}\right) \in \varkappa^{\perp}(N)$, следует, что для $\mathbf{s} \in A(N)$

$$
\Omega\left(2^{-\mathbf{s}}\right) \ll \frac{1}{N(\log N)^{b_{2}-b_{2}^{\prime}}} .
$$

Пусть, далее, $s_{2}<\log N /(6 r)$. Тогда с учетом (42) получаем

$$
\Omega\left(2^{-\mathbf{s}}\right)=\frac{1}{2^{r s_{1}} s_{1}^{b_{1}} 2^{r s_{2}} s_{2}^{b_{2}^{\prime}} s_{2}^{b_{2}-b_{2}^{\prime}}}<\frac{1}{N s_{2}^{b_{2}-b_{2}^{\prime}}} .
$$

Рассмотрим $\sigma_{2}$. С учетом (43) и (44) получим

$$
\begin{aligned}
\sigma_{2} \ll & \left(\sum_{s_{2}<\log N /(6 r), \mathbf{s} \in A(N)}\left(\Omega\left(2^{-\mathbf{s}}\right)\right)^{q_{0}}\right)^{1 / q_{0}} \\
& +\left(\sum_{s_{2} \geqslant \log N /(6 r), \mathbf{s} \in A(N)}\left(\Omega\left(2^{-\mathbf{s}}\right)\right)^{q_{0}}\right)^{1 / q_{0}} \\
& \ll\left(\sum_{s_{2}=1}^{[\log N /(6 r)-1]}\left(\frac{1}{N s_{2}^{b_{2}-b_{2}^{\prime}}}\right)^{q_{0}}\right)^{1 / q_{0}}+\frac{1}{N(\log N)^{b_{2}-b_{2}^{\prime}}}(\log N)^{1 / q_{0}} .
\end{aligned}
$$

Собирая воедино оценки для $\sigma_{1}$ и $\sigma_{2}$ и выбирая наибольшее по порядку слагаемое, получим оценку сверху:

$$
E_{Q^{\prime}(N)}\left(H_{q}^{\Omega}\right)_{q} \ll \frac{1}{N} \cdot \begin{cases}1, & b_{2}-b_{2}^{\prime}>1 / q_{0}, \\ (\log \log N)^{1 / q_{0}}, & b_{2}-b_{2}^{\prime}=1 / q_{0}, \\ (\log N)^{1 / q_{0}-\left(b_{2}-b_{2}^{\prime}\right),} & b_{2}-b_{2}^{\prime}<1 / q_{0} .\end{cases}
$$


Если $q=1$ или $q=\infty$, то

$$
E_{Q^{\prime}(N)}\left(H_{q}^{\Omega}\right)_{q} \ll \sum_{\mathbf{s} \in\left(\varkappa^{\prime}\right)^{\perp}(N)} \Omega\left(2^{-\mathbf{s}}\right)
$$

и мы аналогично получаем оценку (45) с $q_{0}=1$.

По условию теорем $5,6 b_{2}>r+1 / q_{0}$, а при $q=\infty b_{2}>r+1$. Следовательно, можно взять $b_{2}^{\prime}$ такое, что $b_{2}-b_{2}^{\prime}>1 / q_{0}$, и из (45) имеем

$$
E_{Q^{\prime}(N)}\left(H_{q}^{\Omega}\right)_{q} \ll \frac{1}{N}
$$

Отсюда, учитывая неубывание $L_{p}$-нормы по параметру $p$, получаем оценку сверху:

$$
E_{Q^{\prime}(N)}\left(H_{q}^{\Omega}\right)_{p} \leqslant E_{Q^{\prime}(N)}\left(H_{q}^{\Omega}\right)_{q} \ll \frac{1}{N} .
$$

Докажем оценку снизу для $p \leqslant q$. Рассмотрим пачку $\rho(\mathbf{s})$, соответствующую вектору $\mathbf{s}=\left(s_{1}, 1\right), \mathbf{s} \in \theta(N)$. Легко видеть, что $\mathbf{s}=\left(s_{1}, 1\right) \in\left(\varkappa^{\prime}\right)^{\perp}(N)$. Тогда $|\rho(\mathbf{s})| \asymp N^{1 / r}(\log N)^{-b_{1} / r}$.

Как мы знаем, в рассматриваемом случае, $b_{1} \leqslant r, b_{2}>r$, будет

$$
M=|Q(N)| \asymp N^{1 / r}(\log N)^{-b_{1} / r} .
$$

Далее, действуя как при доказательстве примера 1 работы [3], мы можем показать, что существует $\mathbf{k}^{0}=\left(k_{1}^{0}, 1\right) \in \rho\left(s_{1}, 1\right)$ такой, что

$$
\left\|e^{i\left(\mathbf{k}^{0}, \mathbf{x}\right)}-G\left(e^{i\left(\mathbf{k}^{0}, \mathbf{x}\right)}\right)\right\|_{1} \gg 1
$$

Теперь рассмотрим функцию $f\left(x_{1}, x_{2}\right)=C(1 / N) e^{i\left(\mathbf{k}^{0}, \mathbf{x}\right)}$. Функция $f(\mathbf{x})$ принадлежит $H_{q}^{\Omega}$ при некотором $C>0$ для всех $1 \leqslant q \leqslant \infty$. Тогда в силу (47) имеем

$$
\|f(\mathbf{x})-G f(\mathbf{x})\|_{p} \geqslant\|f(\mathbf{x})-G f(\mathbf{x})\|_{1} \gg \frac{1}{N}
$$

что дает требуемую оценку снизу.

Для доказательства оценок снизу при $1 \leqslant q<p \leqslant \infty$ берем функцию, аналогичную функции из (27), однако вместо $\mathbf{s} \in \theta_{1}(N)$ берем $\mathbf{s} \in \theta_{3}(N)$ (см. (23)):

$$
\Phi(\mathbf{x})=C \frac{1}{N} \sum_{\mathbf{s} \in \theta_{3}(N)} 2^{\|\mathbf{s}\|_{1}(1 / q-1)} K_{\mathbf{s}}(\mathbf{x}) .
$$

Теоремы 5 и 6 доказаны. 


\section{Список литературы}

1. Темляков В.Н. Поперечники некоторых классов функций нескольких переменных // ДАН СССР. 1982. Т. 267. № 2. С. 314-317.

2. Темляков B. Н. Приближение функций с ограниченной смешанной производной // Тр. МИАН СССР. 1986. Т. 178. С. 1-112.

3. Темляков $B . H$. Оценки асимптотических характеристик классов функций с ограниченной смешанной производной или разностью // Тр. МИАН СССР. 1989. Т. 189. С. 138-168.

4. Андрианов A.B., Темляков B.H. О двух методах распространения свойств систем функций от одной переменной на их тензорное произведение // Тр. МИРАН. 1997. Т. 219. C. $32-43$.

5. Динь Зунг. Приближение функций многих переменных на торе тригонометрическими полиномами // Матем. сб. 1986. Т. 131(173). № 2(10). С. 251-271.

6. Галеев Э. М. Порядки ортопроекционных поперечников классов периодических функций одной и нескольких переменных // Матем. заметки. 1988. Т. 43. № 2. С. 197-211.

7. Теляковский $C$. A. Некоторые оценки для тригонометрических рядов с квазивыпуклыми коэффициентами // Матем. сб. 1964. Т. 63(105). № 3. С. 426-444.

8. Бари Н. К., Стечкин С.Б. Наилучшие приближения и дифференциальные свойства двух сопряженных функций // Тр. Моск. матем. об-ва. 1956. Т. 5. С. 483-522.

9. Пустовойтов H. Н. Представление и приближение периодических функций многих переменных с заданным смешанным модулем непрерывности // Analysis Mathematica. 1994. T. 20. C. $35-48$.

Поступило в редакцию 2.IV.1998 